\title{
Theoretical Limits for Estimation of Vital Signal Parameters Using Impulse Radio UWB
}

\author{
Sinan Gezici, Member, IEEE, and Zafer Sahinoglu, Senior Member, IEEE
}

\begin{abstract}
In this paper, Cramer-Rao lower bounds (CRLBs) for estimation of vital signal parameters, such as respiration and heart-beat rates, using ultra-wideband (UWB) pulses are derived. In addition, a simple closed-form CRLB expression is obtained for sinusoidal displacement functions under certain conditions. Moreover, a two-step suboptimal solution is proposed, which is based on time-delay estimation via matched filtering followed by least-squares (LS) estimation. It is shown that the proposed solution is asymptotically optimal in the limit of certain system parameters. Simulation studies are performed to evaluate the lower bounds and performance of the proposed solution for realistic system parameters.
\end{abstract}

Index Terms - Ultra-wideband (UWB), impulse radio (IR), Cramer-Rao lower bound (CRLB), least-squares (LS) estimation.

\section{INTRODUCTION}

After the US Federal Communications Commission (FCC) approved the limited use of ultra-wideband (UWB) technology in February 2002 [1], communications and imaging systems that employ UWB signals have drawn considerable attention. A UWB signal is defined to be one that possesses an absolute bandwidth larger than $500 \mathrm{MHz}$ or a fractional (relative) bandwidth larger than $20 \%$. The large bandwidth of UWB signals not only facilitates high-speed data transmission, but also results in high time resolution, which is valuable for precise ranging and location estimation [2], [3].

Commonly, impulse radio (IR) systems, which transmit very short duration pulses with a low duty cycle, are employed to implement UWB systems [4]-[7]. In an IR communications system, a train of pulses is sent and information is usually conveyed by the positions or the amplitudes of the pulses, which correspond to pulse position modulation (PPM) and pulse amplitude modulation (PAM), respectively. In multiuser environments, in order to prevent catastrophic collisions among pulses of different users and thus provide robustness against multiple access interference, each information symbol can be represented by a sequence of pulses and the positions of the pulses within that sequence are determined by a pseudorandom time-hopping (TH) sequence specific to each user [4].

In addition to communications applications, IR-UWB systems have also been considered for medical applications [8][10]. Specifically, high spatial resolution of UWB signals facilitates detection of objects and estimation of vital signal

\footnotetext{
${ }^{0}$ Sinan Gezici is with the Department of Electrical and Electronics Engineering, Bilkent University, Bilkent, Ankara TR-06800, Turkey, Tel: +90 (312) 290-3139, email: gezici@ee.bilkent.edu.tr

Zafer Sahinoglu is with Mitsubishi Electric Research Labs, 201 Broadway, Cambridge, MA 02139, USA, Tel: (617) 621-7588, Fax (617) 621-7550, email: zafer@merl.com.
}

parameters, such as respiration and heart-beat rates of humans, in an environment. Estimation of vital signal parameters can be very important in many scenarios, such as searching people under debris after an earthquake, through-the-wall health monitoring of hostages, and non-invasive patient monitoring [8].

Compared to the Doppler based techniques for estimation of vital signal parameters [11], [12], IR-UWB signalling has advantages such as good penetration capability, which facilitates through-the-wall applications, and low transmission power. In [9], possible medical applications of UWB radars are studied, and their penetration and reflection properties are investigated. In [10], respiratory detection of hidden humans using UWB signals is implemented. A mathematical framework for estimation of vital signal parameters is established in [8], which models IR-UWB signals and related motions filters for estimation of respiration and heart-beat rates.

Although estimation of vital signal parameters using UWB signals have been studied [8]-[10], no studies have considered the theoretical limits and optimal solutions for this parameter estimation problem. In this paper, we derive Cramer-Rao lower bounds (CRLBs) for estimation of vital signal parameters, and propose an asymptotically optimal estimator, which is composed of time-delay and least-squares (LS) estimators. We numerically evaluate CRLBs for practical systems, and compare the performance of the proposed algorithm with CRLBs.

The remainder of the paper is organized as follows. In Section II, the signal model used for parameter estimation is presented. In Section III, the maximum likelihood (ML) solution is obtained and CRLBs are derived for generic and sinusoidal displacement functions. In Section IV, a two-step estimator based on matched-filtering and LS estimation is proposed and its optimality properties are investigated. Then, numerical examples and simulation results are presented in Section V, and concluding remarks are made in Section VI.

\section{Signal Model}

Consider a sequence of pulse bursts as shown in Figure 1 and defined as follows:

$$
s(t)=\frac{1}{\sqrt{N}} \sum_{k=0}^{N-1} w\left(t-k T_{b}\right),
$$

where $N$ is the number of bursts, $T_{b}$ is the burst period, and $w(t)$ is a burst of pulses, which consists of $M$ pulses and is 


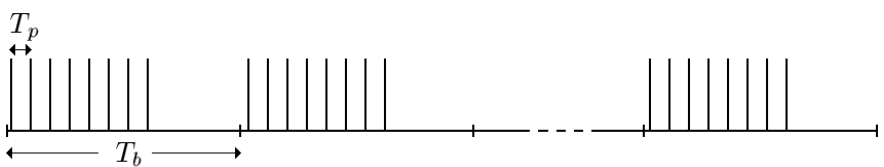

Fig. 1. Transmitted signal structure for estimation of vital signal parameters. Pulses in each burst are used to improve signal-to-noise ratio (SNR) at the receiver, and different bursts give information about vital parameters, such the respiration rate.

expressed as

$$
w(t)=\sum_{j=0}^{M-1} p\left(t-j T_{p}\right),
$$

with $p(t)$ denoting the transmitted pulse and $T_{p}$ being the interval between consecutive pulses. We assume that $T_{p}>T_{\mathrm{w}}$ where $T_{\mathrm{w}}$ denotes the width of $p(t)$.

The burst of pulses in (1) is aimed at an object being monitored and reflections are collected by a receiver. Typically, in pulse-based passive radar applications, pulses in each burst are employed to obtain a reliable channel profile, and comparison of channel profiles obtained from consecutive bursts is used to estimate certain parameters of the target in the environment.

In this paper, we consider a received signal over an additive white Gaussian noise (AWGN) channel with a single path component:

$$
r(t)=g_{\boldsymbol{\theta}}(t)+\sigma_{n} n(t),
$$

where $n(t)$ denotes zero-mean white Gaussian noise with unit spectral density, and

$$
g_{\boldsymbol{\theta}}(t)=\frac{1}{\sqrt{N}} \sum_{k=0}^{N-1} w\left(t-k T_{b}-h_{k}(\boldsymbol{\theta})\right),
$$

with $h_{k}(\boldsymbol{\theta})$ representing the periodic displacement function induced, for example, by respiration or heart-beat of the person being monitored [8], and $\boldsymbol{\theta}=\left[\theta_{1} \cdots \theta_{K}\right]$ denoting the unknown signal parameters. We assume that the range of the displacement function and the pulse $p(t)$ satisfy $(M-1) T_{p}+$ $T_{\mathrm{w}}+\max \left\{h_{k}(\boldsymbol{\theta})\right\}-\min \left\{h_{k}(\boldsymbol{\theta})\right\}<T_{b}$, so that there is no overlap between consecutive pulse bursts, which is usually the case in practical situations.

Although the signal model in (3) is not very realistic for wideband pulse-based systems, it is an important first step towards understanding of a real system since the main ideas in the analysis can be extended to multipath scenarios. Also, this model gets more accurate when directional antennas are used for transmission and reception, and/or an efficient clutter removal algorithm [13] is applied before parameter estimation. Finally, the theoretical limits on the accuracy of parameter estimation obtained using the single path model in (3) provides a lower bound for the multipath case as well, since more nuisance parameters exist for the latter.

\section{MAXimum Likelihood Solution AND CRLB Calculations}

When the signal $r(t)$ in (3) is observed over a time interval $[0, T]$, with $T=N T_{b}$, the log-likelihood function of $\boldsymbol{\theta}$ can be expressed as

$$
\Lambda(\boldsymbol{\theta})=c-\frac{1}{2 \sigma_{n}^{2}} \int_{0}^{T}\left|r(t)-g_{\boldsymbol{\theta}}(t)\right|^{2} d t
$$

where $c$ denotes a constant independent of $\boldsymbol{\theta}$.

Considering real signals, the ML solution can be obtained from (5) as

$$
\hat{\boldsymbol{\theta}}_{\mathrm{ML}}=\arg \max _{\boldsymbol{\theta}} \int_{0}^{T} r(t) g_{\boldsymbol{\theta}}(t) d t
$$

which requires exhaustive search over the parameter space for $\theta$.

From (5), the components of the Fisher information matrix (FIM) $\mathbf{I}_{\boldsymbol{\theta}}$ can be obtained as follows [14]:

$$
\left[\mathbf{I}_{\boldsymbol{\theta}}\right]_{i i}=\mathrm{E}\left\{\left(\frac{\partial \Lambda(\boldsymbol{\theta})}{\partial \theta_{i}}\right)^{2}\right\}=\frac{1}{\sigma_{n}^{2}} \int_{0}^{T}\left(\frac{\partial g_{\boldsymbol{\theta}}(t)}{\partial \theta_{i}}\right)^{2} d t,
$$

for $i=1, \ldots, K$, and

$$
\left[\mathbf{I}_{\boldsymbol{\theta}}\right]_{i j}=\mathrm{E}\left\{\frac{\partial \Lambda(\boldsymbol{\theta})}{\partial \theta_{i}} \frac{\partial \Lambda(\boldsymbol{\theta})}{\partial \theta_{j}}\right\}=\frac{1}{\sigma_{n}^{2}} \int_{0}^{T} \frac{\partial g_{\boldsymbol{\theta}}(t)}{\partial \theta_{i}} \frac{\partial g_{\boldsymbol{\theta}}(t)}{\partial \theta_{j}} d t
$$

for $i \neq j$.

From (4), (7)-(8) can be expressed, after some manipulation, as

$$
\begin{aligned}
& {\left[\mathbf{I}_{\boldsymbol{\theta}}\right]_{i i}=\frac{\tilde{E}}{N \sigma_{n}^{2}} \sum_{k=0}^{N-1}\left(\frac{\partial h_{k}(\boldsymbol{\theta})}{\partial \theta_{i}}\right)^{2},} \\
& {\left[\mathbf{I}_{\boldsymbol{\theta}}\right]_{i j}=\frac{\tilde{E}}{N \sigma_{n}^{2}} \sum_{k=0}^{N-1} \frac{\partial h_{k}(\boldsymbol{\theta})}{\partial \theta_{i}} \frac{\partial h_{k}(\boldsymbol{\theta})}{\partial \theta_{j}}}
\end{aligned}
$$

where $\tilde{E}$ is the energy of the first derivative of the pulse burst $w(t)$; i.e.,

$$
\tilde{E}=\int_{-\infty}^{\infty}\left(\frac{d w(t)}{d t}\right)^{2} d t=M \int_{-\infty}^{\infty}\left(\frac{d p(t)}{d t}\right)^{2} d t
$$

Then, the CRLB for the covariance of an unbiased estimate of $\boldsymbol{\theta}$ can be expressed as [14]

$$
\operatorname{Cov}\{\hat{\boldsymbol{\theta}}\} \geq \mathbf{I}_{\boldsymbol{\theta}}^{-1},
$$

where $\mathbf{B} \geq \mathbf{C}$ means that $\mathbf{B}-\mathbf{C}$ is positive semi-definite.

Let $\theta_{1}=f$ denote the rate (frequency) parameter to be estimated (e.g., respiration rate). Then, the CRLB for estimating $f$ can be stated as

$$
\operatorname{Var}\{\hat{f}\} \geq\left[\mathbf{I}_{\boldsymbol{\theta}}^{-1}\right]_{11}
$$

where $[\mathbf{B}]_{i j}$ denotes the element of matrix $\mathbf{B}$ in the $i$ th row and $j$ th column. 


\section{A. Sinusoidal Displacement Function}

For the remainder of this section, we model the displacement function $h_{k}(\boldsymbol{\theta})$ to have three unknown parameters; frequency $f$, phase $\phi$ and time shift $A$ corresponding to maximum displacement from nominal position. In other words, $\boldsymbol{\theta}=$ $[f \phi A]$. The main parameter of interest is the frequency of the displacement function, which corresponds, for example, to respiration or heart-beat rate of a human. The phase parameter is another unknown since the initial position of the object (e.g., chest cavity) is not known by the receiver. Also, the time shift for the maximum displacement amount from the nominal object position, $A$, is commonly unknown. Since the frequency is the main parameter of interest, the phase and the maximum displacement parameters, $\phi$ and $A$, are treated as nuisance parameters.

For the special case of a displacement function modelled by a sinusoidal function [8], $h_{k}(\boldsymbol{\theta})$ is given by

$$
h_{k}(\boldsymbol{\theta})=A \sin \left(2 \pi f k T_{b}+\phi\right) .
$$

From (14), (9) and (10), the CRLB in (12) can be obtained as

$$
\begin{gathered}
{\left[\mathbf{I}_{\boldsymbol{\theta}}^{-1}\right]_{11}=\frac{N \sigma_{n}^{2}}{2 \pi^{2} T_{b}^{2} A^{2} \tilde{E}}\left(X_{22} X_{33}-X_{23}^{2}\right)\left[X_{11}\left(X_{22} X_{33}-X_{23}^{2}\right)\right.} \\
\left.-X_{12}\left(X_{12} X_{33}-X_{23} X_{13}\right)+X_{13}\left(X_{12} X_{23}-X_{22} X_{13}\right)\right]^{-1},
\end{gathered}
$$

where

$$
\begin{aligned}
& X_{11}=\sum_{k=0}^{N-1} k^{2}\left(1+\cos \psi_{k}\right), \\
& X_{22}=\sum_{k=0}^{N-1}\left(1+\cos \psi_{k}\right), \\
& X_{33}=\sum_{k=0}^{N-1}\left(1-\cos \psi_{k}\right), \\
& X_{12}=\sum_{k=0}^{N-1} k\left(1+\cos \psi_{k}\right), \\
& X_{13}=\sum_{k=0}^{N-1} k \sin \psi_{k}, \\
& X_{23}=\sum_{k=0}^{N-1} \sin \psi_{k},
\end{aligned}
$$

with $\psi_{k}=4 \pi f k T_{b}+2 \phi$.

Although the exact CRLB can be obtained from (15) and (16), a simpler and more intuitive expression can be obtained for the case of a large number of received pulse bursts $N$. The following result can be used to obtain an approximate CRLB expression for large $N$.

Proposition 1: Assume that $T_{b}<\frac{1}{4 f}$. Then, as $N \longrightarrow \infty$,

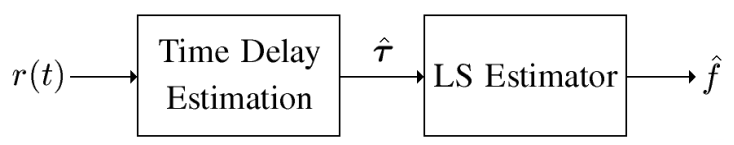

Fig. 2. A two-step suboptimal solution for estimation of vital signal parameters.

the CRLB for $f$ multiplied by $N^{2}$ is expressed as

$$
\lim _{N \rightarrow \infty} N^{2}\left[\mathbf{I}_{\boldsymbol{\theta}}^{-1}\right]_{11}=\frac{6 \sigma_{n}^{2}}{\pi^{2} \tilde{E} A^{2} T_{b}^{2}} .
$$

Proof: See Appendix A.

Note that, in practice, $1 / T_{b}$ is selected to be larger than twice the maximum possible frequency of the displacement function. For example, in [8], for respiration rate estimation, $T_{b}$ is chosen to be 0.1 second, which corresponds to $1 / T_{b}$ being about 10 times the common value for $f$. Therefore, the assumption of $T_{b}<\frac{1}{4 f}$ is valid in most practical situations.

For large $N$, the CRLB for $f$ can be approximated, from (17), as

$$
\left[\mathbf{I}_{\boldsymbol{\theta}}^{-1}\right]_{11} \approx \frac{6 \sigma_{n}^{2}}{\pi^{2} \tilde{E} A^{2} T_{b}^{2} N^{2}} .
$$

From (18), it is observed that as the maximum time displacement amount $A$ or the number of bursts $N$ increases, the minimum achievable variance decreases with the square of these terms. In addition, $\tilde{E} / \sigma_{n}^{2}$ is inversely proportional to the CRLB. It is also important to note from (18) that the approximate expression for the minimum achievable accuracy is independent of the true frequency and phase values. In other words, for any frequency satisfying $f<1 /\left(4 T_{b}\right)$ and any phase, the achievable accuracy is the same for given $A, T$ $\left(T=N T_{b}\right)$ and $\tilde{E} / \sigma_{n}^{2}$ values.

\section{Suboptimal Solution}

Now that we have obtained the best achievable accuracy for frequency estimation, we next consider the problem of obtaining a practical solution that (asymptotically) achieves this best accuracy. Note that the ML solution in (6) is an optimal solution; however, it requires correlation of the received signal over the observation interval $[0, T]$ with the template signal $g_{\boldsymbol{\theta}}(t)$ for various values of the parameter $\boldsymbol{\theta}$, which has prohibitive complexity for practical implementations.

Instead of the ML solution in (6), we propose the solution described in Figure 2. Namely, from $M$ pulses in each burst, we estimate the delay of the signal in the burst. Let $\tau_{k}$ denote the delay of the $k$ th burst. Note that $\tau_{k}$ can be estimated by the conventional TOA estimation algorithm based on matched filter or correlator outputs [15], [16].

Let $\hat{\tau}=\left[\begin{array}{llll}\hat{\tau}_{0} & \hat{\tau}_{1} \cdots \hat{\tau}_{N-1}\end{array}\right]$ denote the delay estimates obtained from matched filter processing. Then, these delay estimates are used by an LS estimator as shown in Figure 2 
to estimate the unknown parameters:

$$
\hat{\boldsymbol{\theta}}=\arg \min _{\boldsymbol{\theta}}\left\{\sum_{k=0}^{N-1}\left(\hat{\tau}_{k}-h_{k}(\boldsymbol{\theta})\right)^{2}\right\} .
$$

Note that this two-step algorithm is considerably simpler and more practical than the ML solution in (6) since it does not have to perform correlations over the whole observation interval for all different values of $\boldsymbol{\theta}$. Instead, it first performs a search over a single dimension, namely time delay, using the matched filter outputs for each given burst. Then, it uses these time delay estimates from different bursts for the LS estimation of the desired parameter(s).

The proposed two-step algorithm is a suboptimal solution in general. However, we now prove that this two-step solution is asymptotically optimal in that it has the same CRLB as in that in Section III, under certain conditions. Towards this end, we first consider the following result:

Lemma 1 [17]: Consider the signal model

$$
\tilde{r}(t)=\frac{1}{\sqrt{N}} w\left(t-\tau_{k}\right)+\sigma_{n} n(t), \quad t \in\left[0, T_{b}\right],
$$

where $n(t)$ is zero mean white Gaussian noise with unit spectral density, and $\tau_{k}$ is the time-delay to be estimated. If $\tau_{k}$ is estimated at a matched-filter output with large SNR. $\beta^{2}$, where $\beta^{2}=\int f^{2}|W(f)|^{2} d f / \int|W(f)|^{2} d f$, it can be modelled as

$$
\hat{\tau}_{k}=\tau_{k}+n_{k},
$$

where $n_{k}$ is a Gaussian random variable $\mathcal{N}\left(0, \sigma_{0}^{2}\right)$ with $\sigma_{0}^{2}=$ $1 /\left(4 \pi^{2} \beta^{2} \mathrm{SNR}\right)$.

In other words, Lemma 1 states that for signals with large SNR and/or effective bandwidth $\beta$, the error of the conventional time-delay estimation can be modelled by a zero mean Gaussian random variable.

According to the two-step solution illustrated in Figure 2, the received signal $r(t)$ in (3) is first used to estimate timedelays $\tau_{k}, k=0,1, \ldots, N-1$. More specifically, $r(t)$ for $t \in$ $\left[(k-1) T_{b}, k T_{b}\right]$ is used for estimating $\tau_{k}$ using a conventional matched-filter approach. For large SNR. $\beta^{2}$, these time-delay estimates are modelled, according to Lemma 1, as

$$
\hat{\tau}_{k}=h_{k}(\boldsymbol{\theta})+n_{k},
$$

for $k=0,1, \ldots, N-1$, with $n_{k} \sim \mathcal{N}\left(0, \sigma_{0}^{2}\right)$. Then, the following proposition states the optimality property of the twostep algorithm in Figure 2.

Proposition 2: For a given set of time-delay measurements $\hat{\tau}=\left[\hat{\tau}_{0} \hat{\tau}_{1} \cdots \hat{\tau}_{N-1}\right]$ modelled by (22), the CRLB for the covariance matrix of an unbiased estimate of $\boldsymbol{\theta}$ is the same as the expression in (12), where the FIM is given by (9)-(11).

Proof: See Appendix B.

Note that the LS estimator in (19) can be shown to be the ML estimator for $\boldsymbol{\theta}$ according to the signal model in (22). Since the ML estimator asymptotically achieves the CRLB [14], which is equal to the CRLB in Section III according to Proposition 2, the LS solution in (19) provides

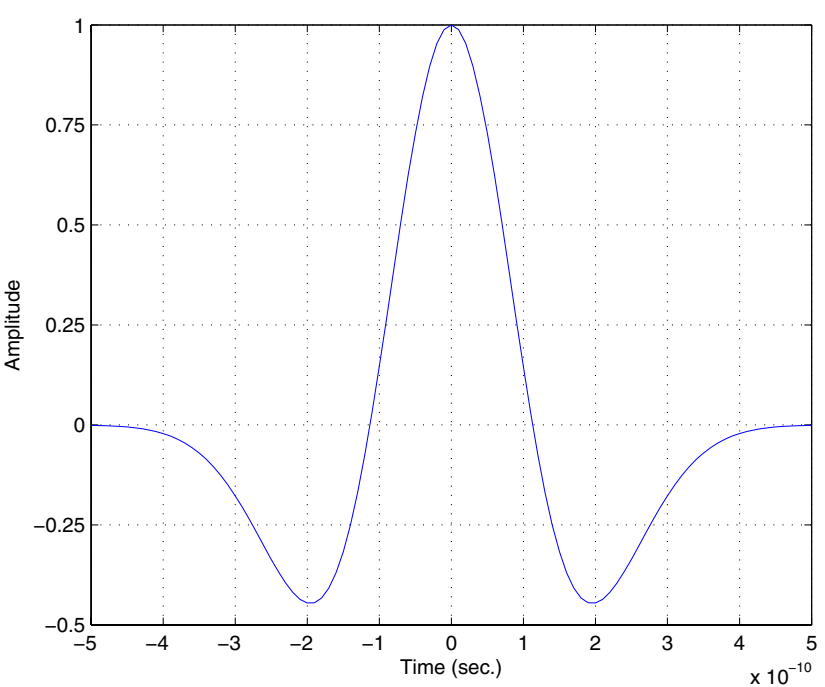

Fig. 3. A UWB pulse with $T_{\mathrm{w}}=1 \mathrm{~ns}$.

an asymptotically optimal solution for estimating $\boldsymbol{\theta}$ under the conditions stated in Lemma 1.

\section{Simulation Results}

In this section, numerical studies and simulations are performed to evaluate the exact and approximate CRLB expressions derived in Section III, and to investigate the performance of the suboptimal solution proposed in Section IV.

Consider a system that uses the following Gaussian monocycle [18]

$$
p(t)=\left(1-\frac{4 \pi t^{2}}{\zeta^{2}}\right) e^{-\frac{2 \pi t^{2}}{\zeta^{2}}} / \sqrt{E_{p}},
$$

where $E_{p}$ is used to adjust the energy of the pulse in the simulations, and $\zeta$ determines the pulse width $\left(T_{\mathrm{w}} \approx 2.5 \zeta\right)$. An example pulse with $T_{\mathrm{w}}=1 \mathrm{~ns}$ is shown in Figure 3.

In Figure 4, the exact and approximate CRLB expressions in (15) and (18), respectively, are compared for various SNRs and pulse widths ${ }^{1}$. The system parameters are $T_{b}=0.1 \mathrm{~s}$, $f=1.1 \mathrm{~Hz}, A=0.1 \mathrm{~ns}$., $\phi=0$, and $N=50$. Although the frequency and phase are set to certain values, the results are practically the same for any frequency satisfying $f<2.5 \mathrm{~Hz}$ and any phase. From Figure 4, it is observed that the exact and approximate CRLBs match very closely for all SNR and $T_{\mathrm{w}}$ values. In addition, the accuracy increases as the pulse width is decreased. This is intuitive since higher time resolution results in better localization of the object (e.g., chest cavity). This can be also observed from (18), since shorter pulses have larger $\tilde{E}$ as can be deduced from (11).

In Figure 5, all the parameters are the same as in the previous case, except that 700 pulse bursts are employed as in the experiment in [8]. In this case, the minimum standard

\footnotetext{
${ }^{1}$ Square-roots of the expressions are plotted and the lower bounds are obtained in units of $\mathrm{Hz}$.
} 


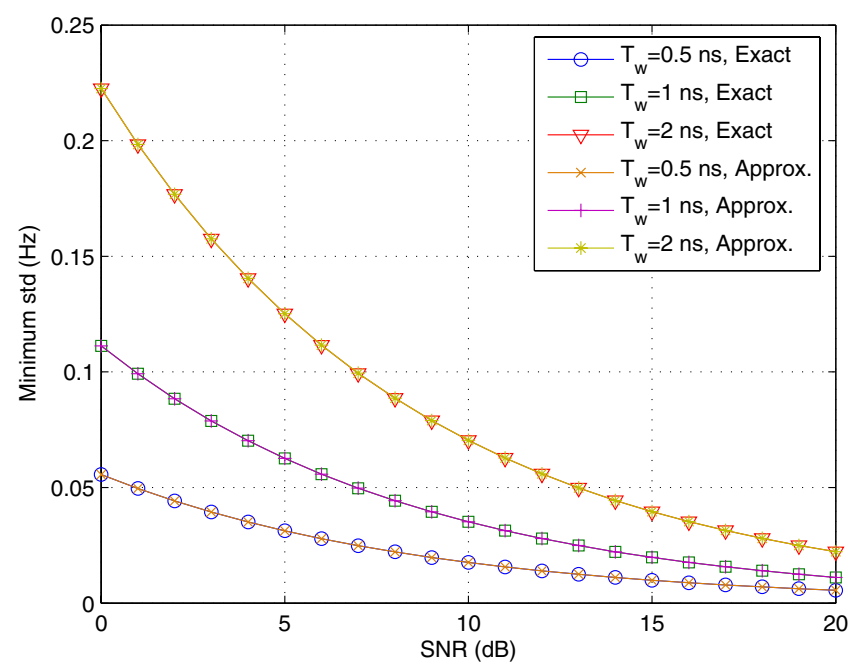

Fig. 4. The exact and approximate CRLB versus SNR for various pulse widths, where $N=50$ bursts are transmitted.

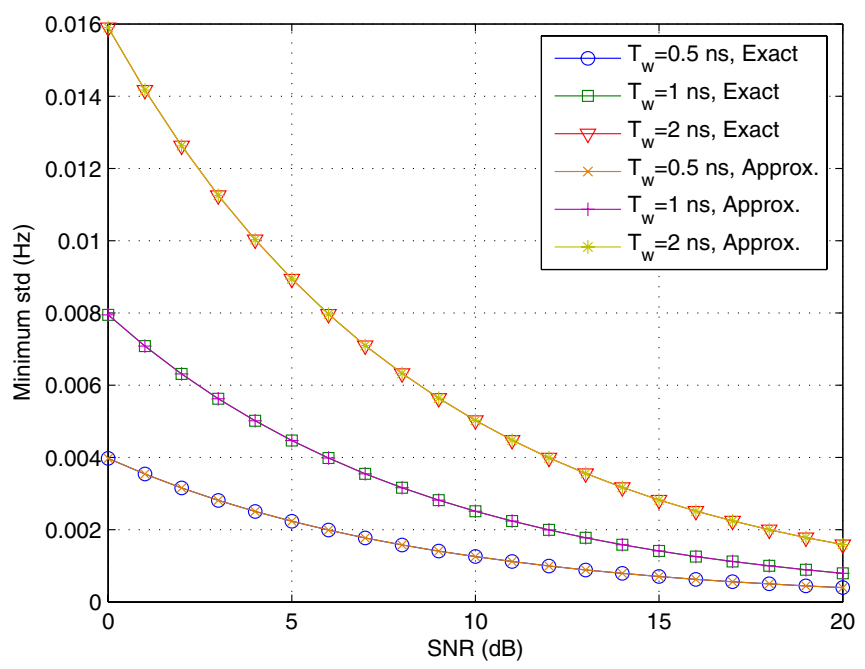

Fig. 5. The exact and approximate CRLB versus SNR for various pulse widths, where $N=700$ bursts are transmitted.

deviations are scaled by 14 compared to Figure 4 , as can be confirmed by (18).

In Figure 6, the same parameters as for Figure 5 are used, and the lower bounds are plotted versus pulse width for various SNRs. It is observed again that as the pulse width decreases, the accuracy of the estimation increases. Moreover, the slope decreases as the SNR increases, which means that for high SNRs, the loss in accuracy when using wider pulses (pulses with smaller bandwidths) is smaller than the loss for low SNRs.

Finally, Figure 7 compares the performance of the suboptimal LS solution in Section IV with the exact and approximate lower bounds for $N=50, T_{\mathrm{w}}=1 \mathrm{~ns}$., $T_{b}=0.1 \mathrm{~s}$., and $A=$

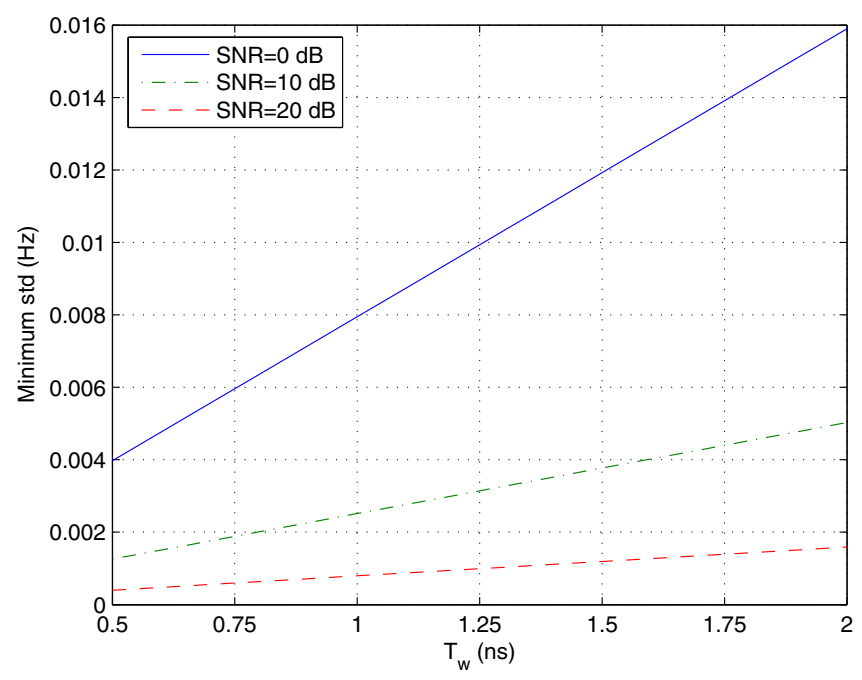

Fig. 6. CRLB versus pulse width for various SNRs, where $N=700$ bursts are transmitted.

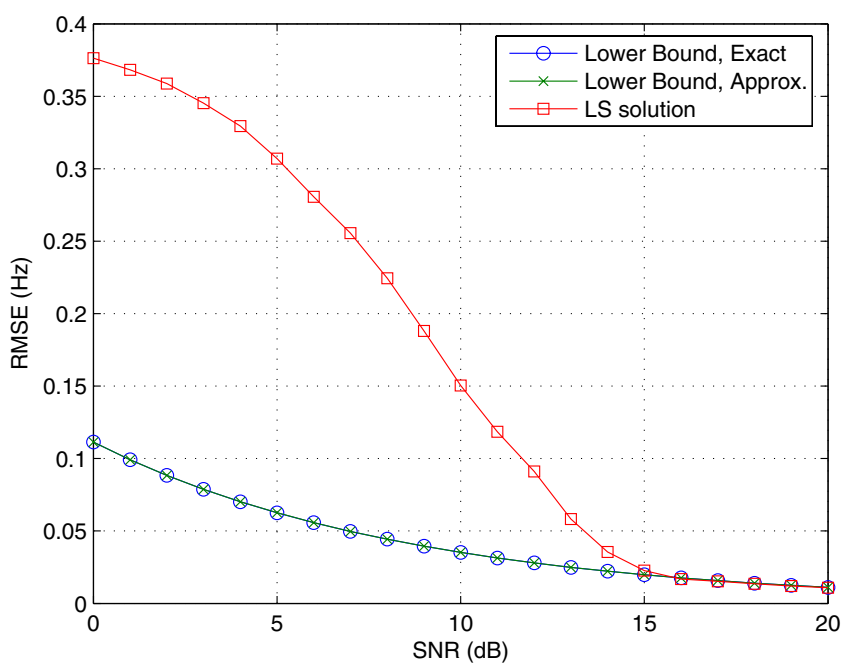

Fig. 7. RMSE versus SNR for the LS solution and the exact and approximate CRLBs for $N=50$ bursts.

0.1 ns. For the LS solution, averages over 10,000 realizations are averaged to calculate root-mean-square errors (RMSEs) for each SNR. For each realization, random noise, $f$ and $\phi$ values are generated. Considering a respiration rate estimation scenario, $f$ is generated randomly from $[0.5,1.5]$. From Figure 7 , it is observed that for large SNR, the performance of the LS solution converges to the lower bound, as expected.

\section{CONCLUSIONS}

Theoretical limits for estimation of vital signal parameters using pulse-based UWB systems have been studied. A generic expression for the CRLB has been obtained for the rate estimation of a periodically moving object. Then, a closedform expression has been derived for the special case of a 
sinusoidal displacement function. Also, a simple and more intuitive approximate bound has been calculated for a large number of pulse bursts in the system. These results not only provide the best achievable limits for rate estimation, but also present guidelines for system design. Finally, in order to have an estimator with good performance and considerably lower complexity than the optimal ML solution, a two-step suboptimal solution has been proposed, which performs matched-filter based time-delay estimations followed by LS estimation.

\section{APPENDIX}

\section{A. Proof of Proposition 1}

Consider the following relation [19]:

$\sum_{k=0}^{N-1} \sin (k y+x)=\sin \left(x+\frac{N-1}{2} y\right) \sin \left(\frac{N y}{2}\right) \operatorname{cosec}\left(\frac{y}{2}\right)$,

with $x=2 \phi$ and $y=4 \pi f T_{b}$.

From (24) and its derivatives with respect to $x$ and/or $y$, closed-form expressions for $X_{11}, X_{22}, X_{33}, X_{12} X_{13}$ and $X_{23}$ in (16) can be obtained ${ }^{2}$. Then, the limit of $N^{2}\left[\mathbf{I}_{\boldsymbol{\theta}}^{-1}\right]_{11}$ as $N \longrightarrow \infty$ can be calculated, after some manipulation, as in Proposition 1.

In the derivations, $\sin (y / 2)$ is assumed to be non-zero. Hence, we considered the case in which $4 \pi f T_{b} / 2<\pi / 2$; i.e., $T_{b}<1 /(4 f)$, as stated in the proposition.

\section{B. Proof of Proposition 2}

From the model in eqn. (22) for the time-delay estimates, the distribution of $\hat{\boldsymbol{\tau}}$ for a given $\boldsymbol{\theta}$ can be expressed as

$$
p_{\boldsymbol{\theta}}(\hat{\boldsymbol{\tau}})=\frac{1}{\left(\sqrt{2 \pi} \sigma_{0}\right)^{N}} \exp \left\{-\frac{1}{2 \sigma_{0}^{2}} \sum_{k=0}^{N-1}\left(\hat{\tau}_{k}-h_{k}(\boldsymbol{\theta})\right)^{2}\right\} .
$$

The elements of the FIM $\tilde{\mathbf{I}}_{\boldsymbol{\theta}}$ can be obtained from

$$
\left[\tilde{\mathbf{I}}_{\boldsymbol{\theta}}\right]_{i i}=\mathrm{E}\left\{\left(\frac{\partial \log p_{\boldsymbol{\theta}}(\hat{\boldsymbol{\tau}})}{\partial \theta_{i}}\right)^{2}\right\}
$$

for $i=1, \ldots, K$, and

$$
\left[\tilde{\mathbf{I}}_{\boldsymbol{\theta}}\right]_{i j}=\mathrm{E}\left\{\frac{\partial \log p_{\boldsymbol{\theta}}(\hat{\boldsymbol{\tau}})}{\partial \theta_{i}} \frac{\partial \log p_{\boldsymbol{\theta}}(\hat{\boldsymbol{\tau}})}{\partial \theta_{j}}\right\},
$$

for $i \neq j$.

From (25), (26) and (27) can be calculated, after some manipulation, as

$$
\left[\tilde{\mathbf{I}}_{\boldsymbol{\theta}}\right]_{i i}=\frac{1}{\sigma_{0}^{2}} \sum_{k=0}^{N-1}\left(\frac{\partial h_{k}(\boldsymbol{\theta})}{\partial \theta_{i}}\right)^{2}
$$

for $i=1, \ldots, K$, and

$$
\left[\tilde{\mathbf{I}}_{\boldsymbol{\theta}}\right]_{i j}=\frac{1}{\sigma_{0}^{2}} \sum_{k=0}^{N-1} \frac{\partial h_{k}(\boldsymbol{\theta})}{\partial \theta_{i}} \frac{\partial h_{k}(\boldsymbol{\theta})}{\partial \theta_{j}},
$$

\footnotetext{
${ }^{2}$ The detailed expressions and the derivations are omitted due to space limitations.
}

for $i \neq j$.

The expression for $\sigma_{0}^{2}$ in Lemma $1, \sigma_{0}^{2}=1 /\left(4 \pi^{2} \beta^{2} \mathrm{SNR}\right)$, can be shown, after some manipulation, to be equivalent to $\sigma_{0}^{2}=N \sigma_{n}^{2} / \tilde{E}$. Hence, (28) and (29) are shown to be equal to (9) and (10), respectively; i.e., $\tilde{\mathbf{I}}_{\boldsymbol{\theta}}=\mathbf{I}_{\boldsymbol{\theta}}$.

\section{REFERENCES}

[1] U. S. Federal Communications Commission, FCC 02-48: First Report and Order.

[2] S. Gezici, Z. Tian, G. B. Giannakis, H. Kobayashi, A. F. Molisch, H. V. Poor and Z. Sahinoglu, "Localization via ultra-wideband radios," IEEE Signal Processing Magazine (Special Issue on Signal Processing for Positioning and Navigation with Applications to Communications), vol. 22, issue 4, pp. 70-84, July 2005.

[3] S. Gezici, Z. Sahinoglu, H. Kobayashi and H. V. Poor, "Ultra wideband geolocation". In H. Arslan, Z. N. Chen and M.-G. Di Benedetto, editors, Ultra Wideband Wireless Communications, Wiley-Interscience, Oct. 2006.

[4] M. Z. Win and R. A. Scholtz, "Impulse radio: How it works," IEEE Communications Letters, 2(2): pp. 36-38, Feb. 1998.

[5] M. Z. Win, R. A. Scholtz, and L. W. Fullerton, "Time-hopping SSMA techniques for impulse radio with an analog modulated data subcarrier," Proc. IEEE Fourth Int. Symp. on Spread Spectrum Techniques \& Applications, pp. 359-364, Mainz, Germany, Sept. 1996.

[6] M. Z. Win and R. A. Scholtz, "Ultra-wide bandwidth time-hopping spread-spectrum impulse radio for wireless multiple-access communications," IEEE Transactions on Communications, vol. 48, no. 4, pp. 679-691, April 2000.

[7] D. Cassioli, M. Z. Win and A. F. Molisch, "The ultra-wide bandwidth indoor channel: from statistical model to simulations," IEEE Journal on Selected Areas in Communications, vol. 20, pp. 1247-1257, August 2002.

[8] S. Venkatesh, C. R. Anderson, N. V. Rivera, and R. M. Buehrer, "Implementation and analysis of respiration-rate estimation using impulsebased UWB," IEEE Military Communications Conference (MILCOM 2005), vol. 5, pp. 3314-3320, Atlantic City, NJ, Oct. 17-20, 2005.

[9] E. M. Staderini "UWB radars in medicine," IEEE Aerospace and Electronic Magazine, vol. 17, no. 1, pp. 13-18, Jan. 2002.

[10] G. Ossberger, T. Buchegger, E. Schimback, A. Stelzer and R. Weigel, "Non-invasive respiratory movement detection and monitoring of hidden humans using ultra wideband pulse radar," IEEE International Workshop on Ultra Wideband Systems (IWUWBS 2004), pp. 395-399, Piscataway, NJ, May 18-21, 2004.

[11] M. Nowogrodzki, D. D. Mawhinney and H. F. Milgazo, "Noninvasive microwave instruments for the measurement of respiration and heart rates," Proc. IEEE National Aerospace and Electronics Conference (NAECON 1984), pp. 958-960, vol. 2, New York, NY, May 21, 1984.

[12] O. B. Lubecke, P. W. Ong and V. M. Lubecke, "10 GHz Doppler radar sensing of respiration and heart movement," Proc. IEEE 28th Annual Northeast Bioengineering Conference, pp. 55-56, Philadelphia, PA, April 20-21, 2002.

[13] M. A. Richards, Fundamentals of Radar Signal Processing, 1st ed. New York: McGraw-Hill, 2005.

[14] H. V. Poor, An Introduction to Signal Detection and Estimation, 2nd ed. New York: Springer-Verlag, 1994.

[15] J. Caffery, Jr., Wireless location in CDMA cellular radio systems, Boston: Kluwer Academic Publishers, 2000.

[16] C. E. Cook and M. Bernfeld, Radar Signal: An Introduction to Theory and Application, Academic Press, 1970.

[17] Y. Qi, "Wireless geolocation in a non-line-of-sight environment," Ph.D. dissertation, Princeton University, Dec. 2003.

[18] F. Ramirez-Mireles and R. A. Scholtz, "Multiple-access performance limits with time hopping and pulse-position modulation," Proc. IEEE Military Communications Conference (MILCOM 98), vol. 2, pp. 529533, Boston, MA, Oct. 1998.

[19] I. S. Gradshteyn and I. M. Ryzhik, Table of Integrals, Series, and Products, 6th ed. SanDiego: Academic Press, 2000. 